Digital Press Social Sciences and Humanities

La rivalité entre de langues des colonisateurs (français et anglais)

Cartalyna Napitupulu

Proceeding of Conférence internationale sur le français 2018

Joesana Tjahjani, Merry Andriani, Sajarwa, Wening Udasmoro (eds) 


\title{
La rivalité entre de langues des colonisateurs (français et anglais)
}

\author{
Cartalyna Napitupulu \\ Universitas Gadjah Mada, Yogyakarta, Indonesia \\ e-mail : cartalynanapitupulu@gmail.com
}

\section{Résumé}

Pendant longtemps le français et l'anglais se rivalisent dans la vie internationale. La mondialisation a conduit à l'internationalisation de l'éducation. Avec le désir des universités de devenir des universités internationales, la position des langues étrangères, en telles que l'anglais, le français, l'allemand, le japonais deviennent plus importante. En France comme dans d'autres pays une des langues les plus importantes est l'anglais. Hagège dit qu'imposer sa langue est aussi imposer sa pensée. Il parait que la France devient plus ouverte à l'anglais. En plus de la croissance des langues étrangères, il est nécessaire de se demander les conséquences de l'internationalisation dans la préservation culturelle, le prestige de la langue nationale, la diversité linguistique et culturelle et la qualité de l'université. Fioraso, l'ancien ministre de l'éducation française, a publié une loi qui est considérée inacceptable pour les syndicats de l'enseignement supérieur. Le 22 mai 2013 des enseignants-chercheurs et étudiants étaient mobilisés, contre le projet de réforme l'enseignement supérieur examiné au pas de charge par l'Assemblée nationale. Enseignants-chercheurs et étudiants étaient mobilisés, mercredi 22 mai, pour protester contre le projet de réforme l'enseignement supérieur examiné au pas de charge par l'Assemblée nationale. En général, les chercheurs qui évaluent l'internationalisation de l'enseignement supérieur ont tendance à partager l'idée que l'internationalisation ouvre des opportunités plus désirables qu'elle ne présente de dangers. Cet article traite l'importance de l'anglais à cause de l'internationalisation de l'éducation en France la domination des Etats Unis. Ces deux langues coloniales continuent à rivaliser et l'utilisation répandue de l'anglais a été rejetée par certaines personnes. Les deux ont un certain prestige, l'anglais est langue de Shakespeare d'un côte et le français est la langue de Molière.

\section{Mots-clés}

langues coloniales, internationalisation de l'éducation, rivaliser

\begin{abstract}
For a long time French and English compete in international life. Globalization has led to the internationalization of education. With the desire of universities to become international universities, the position of foreign languages, such as English, French, German, Japanese become more important. In France as in other countries one of the most important languages is English. Hagège says that to impose one's language is also to impose one's thought. It seems that France is becoming more open to English. In addition to the growth of foreign languages, it is necessary to consider the consequences of internationalization for cultural preservation, the prestige of the national language, linguistic and cultural diversity and the quality of the university. Fioraso, the former French education minister, has published a law that is considered unacceptable for higher education unions. On May 22, 2013 teachers-researchers and students were mobilized, against the project of reform higher education examined at no charge by the National Assembly. Teachers-researchers and students were mobilized on Wednesday, May 22, to protest against the proposed reform of higher education examined at no charge by the National Assembly. In general, researchers who evaluate the internationalization of higher education tend to share the idea that internationalization opens opportunities that are more desirable than dangerous. This article discusses the influence of English, because of the internationalization of education in France and the domination of the United States. These two colonial languages continue to compete and the widespread use of English has been rejected by some people. Both have some prestige, English is Shakespear's tongue of a coast and French is the language of Molière.
\end{abstract}




\section{Keywords}

colonial languages, compete, internationalization of education

\section{Introduction}

A l'époque moderne aucun group social n'est monolingue. En plus de la langue maternelle, chaque individu a une autre langue qui devient sa deuxième langue. On peut devenir multilingue à la suite d'un contact avec son environnement multilingue, et puis maitrise certaine langue grâce à son éducation. Par exemple, une personne qui avait seulement étudié une langue étrangère est devenue plus compétente après avoir appris ou étudié officiellement à l'école.

Une langue peut dominer d'autres langues pour diverses raisons, par exemple pour des raisons économiques, sociales et politiques. Lorsque la France et la Grande-Bretagne contrôlent des pays asiatiques et africains, les habitants des colonies doivent également utiliser la langue des colonisateurs ou de ceux qui sont au pouvoir. Dans les temps modernes, quand le Japon et la Corée se développent économiquement, ces langues aussi commencent à dominer les autres.

Le français et l'anglais sont les deux langues internationales utilisées aux Nations Unies. Ces deux langues se font concurrence pour être dominantes. Les francophones ont tendance à utiliser des termes d'emprunt auxquels s'opposent les nationalistes. Néanmoins, les mots empruntés au français sont également utilisés par les anglophones.

\section{Objectifs}

Cette étude tentera de déterminer dans quelle mesure les deux langues s'influencent et de déterminer les facteurs qui influent sur la domination d'une langue par rapport à une autre. En outre, nous examinerons comment le français maintient sa supériorité sur l'anglais, au contraire comment l'anglais domine le français. De quelle manière les francophones se sentent dominés en anglais et vice versa comment les anglophones se sentent dominés en français.

Cette étude a pour but de voir comment les langues coloniales française et anglaise se sont battues pour leur domination mutuelle et pour les autres langues. En outre, nous examinerons dans quelle mesure les deux langues se dominent et quels facteurs influencent le choix de la langue ou des mots.

\section{Théories}

Les contacts linguistiques ne peuvent pas être évités à l'ère de la mondialisation, qui ont entraînés des changements linguistiques ayant entraîné la création, l'interférence, le changement de code et les mots d'emprunt. Selon Fishman (1991), les groupes des langues minoritaires sont souvent trop faibles pour concurrencer la majorité, de sorte que les langues minoritaires sont marginalisées. On peut dire qu'une langue est faible ou forte en termes de nombre de locuteurs ou en raison de facteurs socio-économiques. Le statut de la langue chinoise est élevé car il compte un grand nombre de locuteurs, alors que le japonais est fort, même si le nombre de locuteurs est faible car il est économiquement fort. Les groupes linguistiques dominants ont la possibilité d'imposer des règles linguistiques et linguistiques aux groupes minoritaires (Hammers \& Blanc, 1989, p. 159). Cette situation entraîne la marginalisation des langues minoritaires.

\section{Analyse}

La Révolution française a introduit le français dans la langue d'enseignement du système éducatif, qui doit être la seule langue d'enseignement. La région qui utilisait à l'origine la langue régionale comme langue d'enseignement a commencé à se tourner vers la langue nationale, à savoir le français. Le français a commencé à jouer un rôle non seulement en France mais également en dehors de la France.

Le gouvernement a également promulgué des lois linguistiques influençant la position de la langue française à l'égard des langues régionales et des langues étrangères. En 1951, le ministre de l'Éducation, Deixonne, promulgua une loi visant à promouvoir l'enseignement des langues régionales dans les écoles. Les nationalistes se sont opposés à cette politique parce qu'elle était considérée comme affectant l'unité 
de la nation. A cette époque, le Conseil de l'enseignement supérieur était encouragé à promouvoir l'utilisation et l'enseignement des langues régionales dans la région (provenant des langues régionales).

En 1975, Pierre Bas et Marc Lauriol ont remplacé la loi Déixonne afin de protéger la France contre la domination des langues étrangères. Toutes les annonces et les annonces doivent être rédigées en français. La loi Toubon de 1994 a également refusé d'utiliser d'autres langues que le français et a encouragé l'utilisation de la langue à l'étranger.

À l'époque moderne, la compétition se déroulait entre l'anglais et le français. Le gouvernement français voit la concurrence mondiale, y compris dans le domaine de l'éducation. L'internationalisation des établissements d'enseignement supérieur entre également dans l'enseignement en France. Constatant cette situation en 2013, le ministre français de l'Education Fioraso a promulgué une loi encourageant l'utilisation de l'anglais dans l'enseignement supérieur. Cela a été rejeté par l'organisation de conférenciers qui a manifesté le 22 mai 2013.

La communauté académique a assisté à cette démonstration. La politique affecte le statut des langues régionales. L'attention du gouvernement est concentrée sur la concurrence avec l'anglais, ce qui laisse de côté l'enseignement des langues régionales. Les ministres Fiorasso du gouvernement socialiste François Hollande pensaient que l'utilisation de l'anglais dans les universités augmenterait la compétitivité des anciens élèves français. L'université internationale de l'université attirera des étudiants étrangers pour étudier en France et pour les étudiants français, les compétences linguistiques en anglais les aideront à faire face à la concurrence économique mondiale.

\subsection{Influences de l'anglais sur le français}

Hagège a adressé diverses critiques à l'anglicisme, notamment l'entrée du vocabulaire anglais en français tels que planning, coach, challenge, life stylecar le français avait un emploi du temps emploi du temps, entraineur, défi, et mode de vie. Le francais n'a pas besoin des mots anglais, ces mots anglais ne forme que des doublets ou ajoute un nouveau vocabulaire. Voir la formation de mots d'emprunt qui suivent la prononciation française comme le mot savoir-faire 'mœurs' remplacé par l'anglais avec la prononciation française "no-'ow" [savoir-faire].

Selon Georges Gastaud, présidentduCollectifUnitaireRépublicain pour la Résistance, l'Initiative et l'EmancipationLinguistique (COURRIEL) 'Unité collective républicaine pour la résistance, l'initiative et l'émancipation linguistiques', la domination de l'anglaissur le français est comme une invasion de la guerre dans divers domaines sociaux sur tout les affaires. En conséquence, les autochtones peuvent devenir étrangers dans leur pays. C'est dangereux parce que le langage est la visiondu monde "Comment regarder le monde". Dans le passé, la domination des langues étrangères avait perturbé l'unité de la nation. Cela pourrait effacer les valeurs héritées des grands penseurs Villon, Rabelais, Molière, Descartes, Diderot, Rousseau, Aragon, Éluard, Césaire et Fanon.

Lespoliticiens et le gouvernement jouent également un rôledans la domination de l'anglais. Christine Lagarde dirige en anglais, Xavier Darcos, ancien ministre de l'Educationsous le règne de SarkozyDarcos, applique efficacement l'anglais à tousles enfants du primaire sous prétexte d'apprendre très tôt les langues étrangères. Si seulement pour cetteraison, il aurait pu suggérer l'allemand, l'espagnol et l'italien pour la régionfrontalière. Valérie Pécresse ministre de l'Enseignement supérieur et de la Recherche du 18 mai 2007 à juin 2011 et la ministredu Budget de mai à mai 2012 ontégalement encouragé l'utilisation de l'anglais dans l'enseignemen tsupérieur (Feltin-Palas, 2012)

Edward (1994, p. 72) affirmeque le choix de la langue ne se faitpas de manièrealéatoire, mais est influencé par desfacteursexternes. La domination de l'anglais en français s'est combinée à l'émergence de la dominationéconomique et militaireaméricaineaprès la 2e Guerre Mondiale. A cette époque on a créel'anglais de base, l'anglaissimplifié qui était l'idée de Charles Kay Ogden en 1925.

Ce modèlelinguistique a réussi à introduirel'anglais en tantque langue de communication. L'anglais a doncétépionnierdans sa position de langue moderne et une langue internationale, et remplace la position dufrançais en tantque langue de commerce, diplomatique et a de nouveauxmotsdans le domaine de la technologie. Dans le monde moderne d'aujourd'hui, l'anglais est la troisième langue la plusparlée et la 
plus étudiée. Margaret Thatcher avaitégalementprédit cela en 2000 (ValèreStaraselski, 2011 dans L'humanité).

Un participantauséminairefrancophonie à Paris de 2006 (Wolton, 2006, p. 129) a déclaréque la protection de la langue françaisedoitcommencerdans le monde desaffaires en appliquantlesloisToubon qui interdisent l'utilisation de languesautresque le français. Dans la société Alcatel oùilstravaillent, ilsdoiventparleranglais. Lesréunions se passaient enanglais. Leslinguistescraignentquecette situation ne menace la position dufrançais.

Claude Hagège défend la diversité des langues et s'oppose à la domination de l'anglais car cette domination constitue une menace pour le patrimoine de l'humanité. Encore plus inquiétant selon lui, la création d'une langue unique encourage la création d'une pensée unique fondée sur le consumérisme et l'argent (Feltin-Palas, 2012)

La domination de l'anglais au français a été critiquée par des linguistes. Le Point fournit des exemples d'entrée du vocabulaire anglais en français. La prédominance de la majorité dans ce cas les domaines économique et technologique a entraîné la marginalisation des langues minoritaires. L'américanisation dans le domaine de la technologie en raison de la popularité et de la force de technologies telles que Google, Yahoo!, L'iPhone et Facebook par les Américains.

Tableu 1 L'anglais remplace le français dans le monde des affaires

\begin{tabular}{|c|c|}
\hline Anglais & Français \\
\hline Hard & Fort, dure \\
\hline Green in les taxis Green Cab G7 & taxis parisiens G7 verts \\
\hline buzz off- rayé du buzz mondial & Décamper \\
\hline
\end{tabular}

Tableu 2 Dans le monde du divertissement

\begin{tabular}{|c|c|}
\hline Anglais & Français \\
\hline Pitches & Ton \\
\hline Cool & Excellent \\
\hline Trendy & Dernier cri, chic \\
\hline
\end{tabular}

Tableu 3 Dans le monde de la mode

\begin{tabular}{|c|c|}
\hline Anglais & Français \\
\hline un jean slim, medium & Moyen \\
\hline Magasinage & Shopping \\
\hline Pressage & Pressing \\
\hline Chandails & sweat-shirts \\
\hline
\end{tabular}

Tableu 4 Dans le monde du tourisme

\begin{tabular}{|c|c|}
\hline Anglais & Français \\
\hline Trip;travel & Voyage \\
\hline Package & Paquet \\
\hline check-in & lecheck-in \\
\hline check-out & le check-out \\
\hline
\end{tabular}




\subsection{Rejet de l'influence de l'anglais}

Parlant de l'influence de la langue anglaise, on connaît le terme d'anglicisme, qui a un caractère anglais. Dans ce cas, il s'agit d'un vocabulaire lié à l'anglais ou le mot français qui a des caractères anglais. Le mot franglais (français anglais) est considéré comme un vocabulaire français avec des caractères anglais. Le vocabulaire a été formé en raison de l'influence de l'anglais sur le français (Lexico, s.d).

Le rejet de la domination de l'anglais a été montré par plusieurs groupes. Un lecteur de BBC News a témoigné qu'il désapprouvait l'influence de l'anglais ou de l'anglicisme par un ingénieur français qui dit know-howà la française /no-'ow/ pour remplacer savoir-faire («Englishisms in France : Readers' franglais favourites», 2013)

Dans les cours de français on entend des mots anglais tels que;les bakedbeans, faire du shopping et le booze-cruising. À la gare anglaise, il y a des mots français comme dans la phrase The buffet isopen. Pendant ce temps en France, l'équivalent est Le snack bar est ouvert. C'est le must ! C'est de rigueur. Les talkies-walkies anglais deviennent des talkies-walkies ! Les français utilisent les expressions cool, supercool et hypercool / eepakul /.

De plus, il existe un phénomène consistant à créer des termes anglais en traduisant l'anglais pour remplacer le terme français couramment utilisé par des anglophones, tels que Good eating!PourBonAppétit! Le français fait aussi de nouveaux mots ou franglais (un mélange d'anglais et de français) à partir d'anglais tels que, un one-man-show, et une one-woman-show. Luncher du mot to lunch. Lunchez-vous avec moi aujourd'hui ?" Veux-tu déjeuner avec moi ? footing 'jogging', home staging 'décoration'

La prédominance de l'anglais se manifeste également par l'utilisation du terme le redcarpetà la place du tapis rouge. D'autres mots hybrides sont smoking, a parking, a dressing", a shampooing", a snacking.Le mot smoking est ambigu, car il peut signifier le type de tenue de soirée.

Agnes Poirier (2013), journaliste française, a écrit en 2003dans le magazine Magazine que l'idée de Fiorasso n'était pas nouvelle. Les grandes écoles, prestigieuse institution tertiaire, animent des conférences en anglais depuis 15 ans.

Le journal de gauche Libération a écrit que 790 collèges avaient donné des conférences en anglais. Il n'avait rien de fausse de la décision de Fiorasso. Les jeunes ont l'habitude d'utiliser le franglais dans leur vie quotidienne et considèrent le travail de l'Académie française n'est plus important.

Les linguistes s'opposent au choix de l'anglais considéré comme pratique comme la langue d'enseignement pour des raisons suivantes.

1. En termes d'écriture; il y a 5 façons de prononce la lettre ou en mots through, rough, bough, four et tour

2. En termes deprécision: l'anglaisestinefficaceet a entraînédesaccidentsd'avion. Le 29 décembre 1972, l'avions'estécraséenFloride, lorsquelatourdeguet a déclaré: Turnleft, rightnow«Tournez à gauche, maintenant», le pilotel'ainterprétécomme« tournez à gauche, tournez à droite ».

3. De même dans le monde de la diplomatie, la résolution $n{ }^{\circ} 242$ de 1967 des Nations Unies sur le retrait des forces armées israéliennes des territoires occupés lors du récent conflit, du" withdrawal of Israel armed forces from territories occupied in the recent conflict" "retrait des forces armées israéliennes de la zone de conflit récemment occupée" Les pays arabes interprètent qu'Israël doit se retirer "du" territoire occupé - sous-entendu - de tous. Alors qu'Israël estime qu'il suffit de se retirer "des" territoires occupés, ce qui signifie qu'une partie seulement du territoire est occupée.

La résistance au français a incité les francophones à former de nouveaux mots pour rejeter l'anglicisme.

Tableu 5 Dans le monde du divertissement

\begin{tabular}{|l|l|}
\hline Français & Pour remplaceranglicisme \\
\hline heure de grandeécoute & prime-time \\
\hline spectacle solo & one-man show \\
\hline film à sensations & thriller \\
\hline
\end{tabular}




\begin{tabular}{|l|l|}
\hline Financeur & sponsor \\
\hline succès de librairie & best-seller \\
\hline Exclusivité & scoop \\
\hline mise à l'index & boycott. \\
\hline
\end{tabular}

Tableu 6 Dans le monde culinaire

\begin{tabular}{|l|l|}
\hline Français & Pour remplaceranglisisme \\
\hline Canneberge & Cranberries \\
\hline pommes de terre & Patate yang berasaldari potato \\
\hline casse-croûtes & Sandwich \\
\hline restaurant rapide, restovite & fast-food \\
\hline
\end{tabular}

Tableu 7 Dans le monde des jeux

\begin{tabular}{|l|l|}
\hline Français & Pour remplaceranglisisme \\
\hline Divulgâcheur & Spoiler \\
\hline casse-tête & Puzzle \\
\hline Smatte & Smart \\
\hline
\end{tabular}

\subsection{Effet du français sur l'anglais}

Isabel Roth (2010) examine l'influence du français sur la langue en prenant des données d'un article de journal dans les deux langues pour examiner les mots empruntés aux périodes du moyen anglais (EM) et du début de la modernité anglaise (EModE), ainsi que leurs effets sur l'affixation. Il a également examiné l'influence de la sociolinguistique.

Le premier contact entre l'anglais et le français eut lieu en 1042, lorsqu'Edward le confesseur exilé en Normandie revint sur le trône. Puis, en 1066, le groupe New Norman régna sur l'Angleterre dans la langue anglo-française ; l'anglais influencé par le français. La langue qu'ils utilisaient a affecté la langue des employés et est devenu la langue du commerce et des tribunaux. Cette situation a rendu la position du français plus élevée et de nombreux mots d'emprunt du français sont entrés en anglais ; comme trône>throne. Le mot Saint du latin au français. Après la période d'occupation, le français était utilisé par l'élite pour entrer dans les classes inférieures et être utilisé quotidiennement.

Vers 1258-1362, le français n'appartenait plus à l'élite, car il était devenu une langue de tous les jours. Il est de plus en plus important de pouvoir parler français dans la classe moyenne. Les communautés linguistiques ont leur propre évaluation de l'existence et de l'utilisation de certaines langues. Il en résulta une langue prestigieuse, privilégiée pour certaines langues et moins favorisée dans les langues considérées comme moins prestigieuses (Wardaugh, 1992). Les langues régionales sont généralement considérées comme moins prestigieuses que les langues officielles. Dans ce cas, l'anglais et le français sont considérés comme prestigieux, car ce sont les langues utilisées lors des réunions officielles de l'ONU.

Lorsque les mots d'emprunt français sont entrés en anglais, il y avait aussi des commentaires obliques de philosophes français qui montraient un sentiment de concurrence entre les deux nations.

Les Anglais ont pris de nous ces mots apointé, désapointé, ainsi que beaucoup d'autres expressions très énergiques : ils se sont enrichies, de nos dépouilles et nous n'osons reprendre notre bien (Steuckardt, 2006).

Tableu 8 Le vocabulaire aristocratique anglais utilise le français

\begin{tabular}{|c|c|}
\hline Français & Anglais \\
\hline Cardinal & Cardinal \\
\hline Prison & Prison \\
\hline
\end{tabular}




\begin{tabular}{|c|c|}
\hline Justice & Justice \\
\hline Courone & Crown \\
\hline Militaire & Military \\
\hline Juridique & Juristic \\
\hline Religieux & Religious \\
\hline Animal & Animal \\
\hline Fruit & Fruit \\
\hline Village & Village \\
\hline
\end{tabular}

Les linguistes français regrettent l'immense domination de l'anglais en français. Mais Anthony Lacoudre (Gaétan, 2015) un bilingue (pèrefrançais et mèreanglaise) résidant à Paris et à New York qui considère de nombreux mots français en anglais. Ceci est rejeté par les anglophones en disant queles mots viennent du latin.

La révolution industrielle a produit de nouveauxmots en français, telsque "turbine", "microbe", "téléphone", photographie ". Desinjures en français, par exemple: crétin, rascal, stupide, bastard,coward et imbécile sont entrés dans le vocabulair eanglais. Cela s'est passé pendant la guerre de cent ans, lorsque des soldats britannique sont négocié avec Jeanne d'Arc. Ils se grondent en français.

Les emprunts du français à l'anglais se passe danslesannées 60. En raison de l'influenceaméricaine, il y a une tendance à l'influence de l'anglaisvers le français. Le derniermotfrançaisentre en anglais est bustier. Les emprunts peuvent faire un aller-retour car le mot people pour parler descélébrités vient du mot people en anglaisau Moyen Âge. Le mot de nice vient du mot français au Moyen Âgesignifie «stupide». La signification du mot peut aussi changer avec le temps, comme le motbacon "viandefumée" qui provient du bacon français "graisse de porccoupée".

\section{Conclusion}

La France a déjà triomphé dans le passé et le français est entré dans le vocabulaire anglais. Mais à l'époque moderne, l'anglais semble cesser d'emprunter des mots français et l'anglais domine davantage. La domination de l'anglais est inévitable compte tenu de la domination de l'Amérique. La dominance des langues est négative si elle fait référence à des monolingues.

Si l'anglais domine en raison de la technologie américaine et de l'américanisation par le biais de la culture hollywoodienne, la France doit compenser en renforçant sa langue comme la langue Molinière qui fait venir de grands penseurs, Éluard, Césaire et Fanon. En effet, la France domine la mode de la classe supérieure avec la haute couture et son monde culinaire. Mais si elle ne touche que la classe supérieure, elle est moins enracinée, de sorte que ce langage perdra de son influence.

La rivalité entre les deux langues se poursuit. Le propriétaire de la langue peut maintenir sa domination en regardant les domaines importants qui constituent la force de sa communauté de langue et qui deviennent le prestige de sa langue. L'anglais est avec l'américanisation dans le domaine de la technologie et du divertissement avec ses films et ses artistes hollywoodiens.

Par ailleurs, la France doit continuer à explorer le potentiel de ses citoyens capables d'équilibrer l'américanisation. L'internationalisation menant au monolingualisme renforcera la domination de l'anglais, car les gens ont tendance à choisir d'utiliser l'anglais. Par conséquent, pour réduire la domination de l'anglais, la France a non seulement encouragé la francophonie, mais également le multilinguisme.

\section{Références}

Englishisms in France: Readers' franglais favourites. (2013). Retrieved from http://www.bbc.com/news/magazine-22636888

Edwards, J. (1992). Multilingualism. London: Routledge. 
Feltin-Palas, M. (2012). Claude Hagège: “Imposer sa langue, c'est imposer sa pensée.” Retrieved from https://www.lexpress.fr/culture/livre/claude-hagege-imposer-sa-langue-c-est-imposer-sapensee_1098440.html

Fishman, J. (1991). Reversing Language Shift: Theoretical and Empirical Foundations of Assistance to Threatened Languages. Clevendon: Multilingual Matters.

Gaétan, M. (2015). "L'influence du français sur la langue anglaise est considérable et insoupçonnée." Retrieved from https://france-amerique.com/en/linfluence-du-francais-sur-la-langue-anglaise-estconsiderable-et-insoupconnee/

Hamers, J. F., \& Blanc, M. H. A. (1989). Bilinguality and Bilingualism Second edition. Cambridge: Cambridge University Press. https://doi.org/10.1017/CB09781107415324.004

Lexico. (n.d.). Englishism. Retrieved from https://en.oxforddictionaries.com/definition/englishism Poirier, A. (2013). Franglais row: Is the English language conquering France?

Roth, I. (2010). Explore the influence of French on English. Innervate: Leading Undergraduate Work in English Studies, 3, 255-262.

Steuckardt, A. (2006). L'emprunt et sa Glosse. Mots. Les Langages Du Politique, (82), 9-22.

Wardaugh, R. (1992). An Introduction to Sociolinguistics. Oxford: Blackwell. https://doi.org/10.2307/590702 